\title{
Phase Diagram of a Model for Diluted Magnetic Semiconductors Beyond Mean-Field Approximations
}

\author{
Gonzalo Alvarez, ${ }^{1}$ Matthias Mayr, ${ }^{2}$ and Elbio Dagotto ${ }^{1}$ \\ ${ }^{1}$ National High Magnetic Field Lab and Department of Physics, Florida State University, Tallahassee, FL 32310 \\ ${ }^{2}$ Max-Planck-Institut für Festkörperforschung, 70569 Stuttgart, Germany.
}

(Dated: November 13, 2018)

\begin{abstract}
A lattice spin-fermion model for diluted magnetic semiconductors (DMS) is investigated numerically, improving on previously used mean-field (MF) approximations. Curie temperatures are obtained varying the Mn-spin $x$ and hole $n$ densities, and the impurity-hole exchange $J$ in units of the hopping amplitude $t$. Optimal values are found in the subtle intermediate regime between itinerant and localized carriers. Our main result is the behavior of the Curie temperature at large $J / t$, where a "clustered" state is observed and ferromagnetism is suppressed. Formal analogies between DMS and manganites are also discussed.
\end{abstract}

PACS numbers: 75.50.Pp, 75.10.Lp, 75.30.Vn

Diluted magnetic semiconductors based on III-V compounds are attracting considerable attention due to their combination of magnetic and semiconducting properties, that may lead to spintronic applications [1, 2 . $\mathrm{Ga}_{1-x} \mathrm{Mn}_{x} \mathrm{As}$ is the most studied of these compounds with a maximum Curie temperature $T_{C} \approx 110 \mathrm{~K}$ at low doping $x$, and with a carrier concentration $p=(n / x)<1$ due to the presence of As antisite defects [1] or Mn intersticials [3]. It is widely believed that this ferromagnetism is carrier-induced, with holes introduced by doping mediating the interaction between $S=5 / 2 \mathrm{Mn}$-spins. This Zener mechanism operates in other materials as well [4.

In spite of the excitement around $110 \mathrm{~K}$ DMS, room temperature ferromagnetism should be achieved for potential applications, with logic and memory operations in a single device. For this reason, a goal of the present effort is to analyze the dependence of $T_{C}$ on the parameters $x, p$, and $J / t$, helping in setting realistic expectations for DMS potential technological applications. This goal can only be achieved with good control over the many-body aspects of the problem, and for this purpose lattice Monte Carlo (MC) techniques are crucial, improving on previously employed MF approximations. Our results lead to an optimistic view in this respect, since $T_{C}$ is found to increase linearly with $x$ up to $x \sim 0.25$.

Our effort builds upon previous important DMS theoretical studies [2, 0, 6, , 8, 8, 9, 10]. However, to analyze whether $T_{C}$ can be substantially increased from current values, techniques as generic as possible are necessary. In particular, both the strong interactions and disorder must be considered accurately, with computational studies currently providing the best available tools. For these reasons, our work differs from previous approaches in important qualitative aspects: (1) Some groups use a continuum six-band description of DMS [5]. (2) Other theories assume carriers strongly bounded to impurity sites [6], and employ Hartree-Fock approximations. (3) Dynamical MF theory (DMFT) [7] may not capture the percolative character of DMS, with a random impurity distribution and cluster picture [8, 9]. (4) Other approaches use MF uniform states [2], or introduce a reduced basis in simulations [10]. While the previous work is important in describing current DMS materials, our goal is to establish the phase diagram of a DMS model avoiding MF approximations.

For the above mentioned reasons, here a generic MC study of a lattice spin-fermion model for DMS is reported. The Hamiltonian is

$$
\hat{H}=-t \sum_{<i j>, \sigma} \hat{c}_{i \sigma}^{\dagger} \hat{c}_{j \sigma}+J \sum_{I} \vec{S}_{I} \cdot \vec{\sigma}_{I}
$$

where $\hat{c}_{i \sigma}^{\dagger}$ creates a hole at site $i$ with spin $\sigma$, and the hole spin operator interacting antiferromagnetically with the localized Mn-spin $\vec{S}_{I}$ is $\vec{\sigma}_{I}=\hat{c}_{I \alpha}^{\dagger} \vec{\sigma}_{\alpha, \beta} \hat{c}_{I \beta}$. The carrier can visit any site of the lattice (assumed cubic [11). The interaction term is restricted to randomly selected sites, $I$, with a $S=5 / 2 \mathrm{Mn}$-moment. These spins are here considered classical with $\left|\vec{S}_{I}\right|=1$, as widely assumed, allowing for a MC simulation similarly as in other contexts [12. Photoemission and band calculations locate $J / t$ near 2 [7, 10, 13, 14. Rather than fixing parameters to current DMS values, here $J / t$ is unrestricted, while $x$ and $p$ mainly vary in the range $[0,1]$. Approximations include the neglect of on-site Coulomb repulsion, valid at small $x$ and $p$ where double occupancy is unlikely. In addition, Mn-oxides investigations [1] show that an intermediate or large $J / t$ plays a role analogous to a Hubbard $U / t$ at any $x$ [1] 15]. At low $x$, the probability of nearestneighbors (NN) Mn-spins is also low (0.0625 at $x=0.25)$, justifying the neglect of an antiferromagnetic (AF) MnMn coupling. The hole motion is described by a oneband tight-binding model, while a more realistic model should include many bands as well as spin-orbit interaction [5]. Despite this simplification, our study considers the underlying lattice, absolutely necessary for a qualitative understanding of the DMS phase diagram.

The MC technique used here is as in Mn-oxides investigations [4]: it includes the full Exact Diagonalization (ED) of the hole sector for each MC spin configuration, and density-of-states expansion calculations 

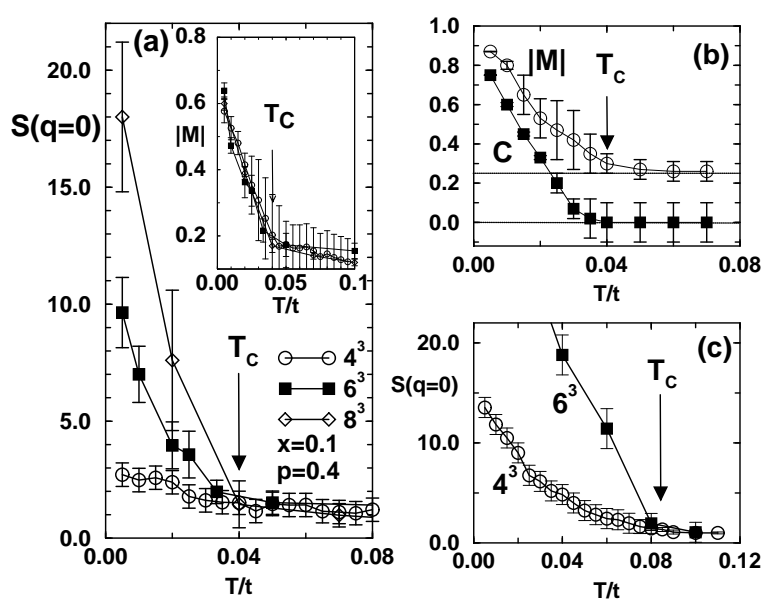

FIG. 1: (a) $S(q=0)$ vs. temperature, $T$, for $4^{3}$ (circles), $6^{3}$ (squares), and $8^{3}$ (diamonds) clusters at $J / t=2.0, x=0.1$ and $p=0.4$, using the MC technique. The inset shows the magnetization $|M|$ for the same clusters, with a vertical scale referred to the asymptotic $8^{3} T=\infty$ value. (b) $|M|$ (circles) and spinspin correlation $C$ at maximum distance (squares) vs. $T$, on a $4^{3}$ cluster, at $J / t=1.0, x=0.25$, and $p=0.4$. The 0.25 horizontal line indicates the $|M| T=\infty$ asymptotic value. (c) $S(q=0)$ vs. $T$, at $J / t=2.0, x=0.25$, and $p=0.4$ using $4^{3}$ (circles) and $6^{3}$ (squares) clusters. In all cases $T_{C}$ is indicated.

[16]. The latter allows us to reach clusters with up to $8^{3}=512$ sites if up to 40 terms are included, reaching an accuracy comparable to ED for smaller clusters. Both methods are nearly exact, and the error bars of our results mainly arise from intrinsic thermal fluctuations and averages over several random Mn-disorder configurations. Comparing estimations of different clusters and based on previous experience with similar models [4], $T_{C}$ can be calculated within a $\sim 25 \%$ accuracy, sufficient for our purposes [17]. The order parameter for the ferromagnetic-paramagnetic transition was taken to be the absolute value of the magnetization of the Mnspins normalized to 1 , namely $\left.|M|=\frac{1}{x N} \sqrt{\sum_{I, R}\left\langle\vec{S}_{I} \cdot \vec{S}_{R}\right.}\right\rangle$. Size effects are better visualized in the zero-momentum spin structure factor $S(q=0)=\frac{1}{x N} \sum_{I, R}\left\langle\vec{S}_{I} \cdot \vec{S}_{R}\right\rangle$. Another useful quantity is the spin-spin correlation at distance $d$, $C(d)=\frac{1}{N(d)} \sum_{|I-R|=d}\left\langle\vec{S}_{I} \cdot \vec{S}_{R}\right\rangle$, where $N(d)$ is the number of pairs of Mn moments separated by a distance $d$.

Typical results for small and intermediate $J / t$ of our large-scale computational effort are in Fig.1a. There $S(q=0)$ and $|M|$ vs. temperature $T$ are shown for three cluster sizes, $x=0.1$, and $p=0.4$. Note the small size dependence of the magnetization (inset), and the volume growth of $S(q=0)$ at fixed $T<T_{C}$. The estimated $T_{C} / t$ is $\sim 0.04$, with an uncertainty 0.01 sufficiently small for our purposes. Even with just the $4^{3}$ cluster, $T_{C}$ could be estimated fairly well, as shown in Fig.1b. This is important to simplify our computational search for optimal $T_{C}$ 's varying many parameters. In Fig.1b, the temperature where a deviation from the high- $T$ limit is found is slightly larger than the $T_{C} / t=0.04$ obtained from larger clusters (indicated). Studying the spin-spin correlation at the largest available distance, a nonzero value characteristic of an ordered ferromagnetic (FM) state was obtained at $T$ just below 0.04 . Figure $1 \mathrm{c}$ provides another example of our comprehensive $T_{C}$ study, using just two cluster sizes at the $x-p$ location of our most optimal $T_{C}$, at fixed $J / t=2$. Here the use of only $4^{3}$ and $6^{3}$ clusters provides once again a fairly accurate value $T_{C} \sim 0.08 t$.

To understand the qualitative $T_{C}$ trends, first consider the simplest case: the $p$ dependence at fixed $J$ and $x$. Using the results in Fig.2a contrasted against Fig.1b (same cluster size) $T_{C}$ is found to change by a factor $\sim 2$, when $p$ varies from 0.1 to 0.4 . However, this tendency does not continue with increasing $p$, since at $p=1$ or beyond, a FM state is not formed: the Pauli principle reduces drastically the carrier kinetic energy, leading instead to an $\mathrm{AF}$ state. An example at $p=3$ and on an $8 \times 8$ cluster (results are qualitatively similar in two and three dimensions) is in Fig.2b, where the oscillations in the spin correlations indicate staggered order. In general, the optimal $p$ is $\sim 0.5$, between the hole empty $p=0$ and saturated $p=1$ limits, as found with DMFT [7]. A similar result occurs in Mn-oxide models, recovered from Eq.(1) at $x=1$. In that context, investigations at large Hund coupling, the analog of $J$ for DMS, have shown that $p=0.5$ optimizes $T_{C}$ to a number $\sim 0.11-0.13 t$ [4, 18], likely an upper-bound on the $T_{C}$ that could be achieved with Eq.(1).
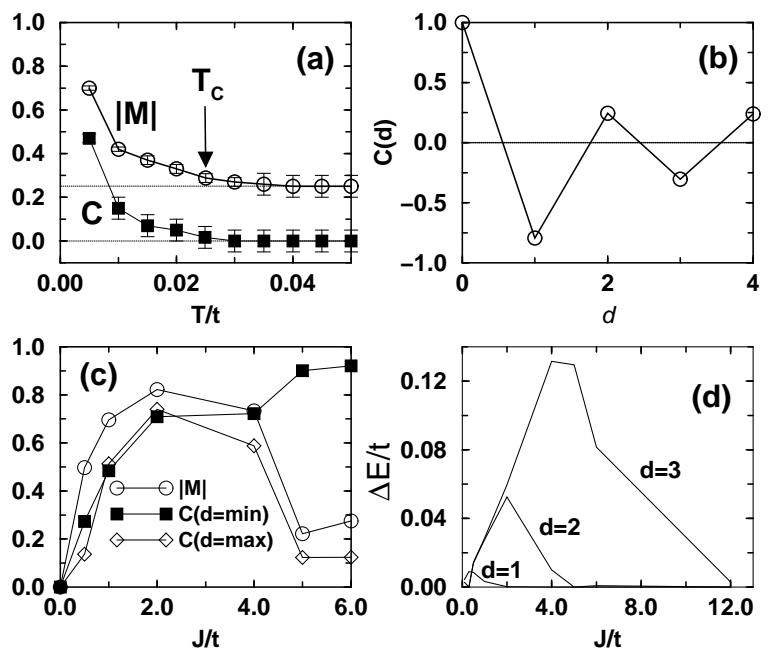

FIG. 2: (a) Magnetization $|M|$ (circles), and spin-spin correlation at maximum distance $C$ (squares) vs. $T$, at $J / t=1.0$, $x=0.25$, and $p=0.1$, using a $6^{3}$ cluster. (b) $C(d)$ vs. $d$ at $p \sim 3.0, x=0.25, J / t=1.0$, and $T / t=0.005$, using an $8^{2}$ cluster. The oscillations indicate an AF state. (c) $|M|$ (circles), spinspin correlation at minimum distance $C\left(d_{\text {min }}\right)$ (squares), and at maximum distance $C\left(d_{\max }\right)$ (diamonds) vs. $\mathrm{J} / t$, for a $4^{3}$ cluster at $x=0.25, p=0.1$, and $T / t=0.005$. (d) $\Delta E=E(\theta=\pi)$ $E(\theta=0)$ vs. $J / t$ calculated exactly on finite but large clusters at $T=0$ for a system of $2 \mathrm{Mn}$-spins and 1 electron, $\theta$ being the relative angle between the $\mathrm{Mn}$-spins. Results in 1,2 , and 3 dimensions are indicated. The spin distance is such that the associated effective $x \sim 0.1$ is the same in all cases. 
Consider now the $J / t$ dependence of $T_{C}$. The MF approximation suggests $T_{C}^{m f} \propto J^{2}$. However, this does not hold when more accurate methods are used in the calculations. In fact, for $J / t \rightarrow \infty$ and a Mn dilute system, the holes are trapped in Mn-sites, reducing drastically the conductance and $T_{C}$. Small FM clusters of spins are formed at a temperature scale $T^{*}$, but there is no correlation between them, leading to a global vanishing magnetization [9]. These results cannot be obtained within a mean-field approximation. The large- $J / t$ ideas can be tested in our MC simulation by monitoring the short- and long-distance behavior of the spin-spin correlations $C(d)$. In a "clustered" state (large $J / t), C(d)$ at the shortest distance can be robust at $T<T^{*}$, but $C(d)$ at the largest distance vanishes due to the uncorrelated nature of the magnetism between independent clusters (see Fig.2c). This subtle effect explains the incorrect MF prediction, since $T_{C}^{m f} \sim T^{*}$, which grows with $J / t$, rather than the true $T_{C}$ (see also Fig.4a). Since both in the $J / t \sim 0$ and $J / t=\infty$ limits $T_{C}$ is suppressed, an optimal $J /\left.t\right|_{\text {opt }}$ must exist where $T_{C}$ is maximized. Simulation results as in Fig.2c indicate that the optimal $J / t$ value is close to 2. This phenomenon is not captured in itinerant [2] or localized [6] limits nor by DMFT [7], but it is observed in the present generic MC simulations.

The existence of a $J /\left.t\right|_{\text {opt }}$ can be illustrated just using two spins and one carrier in a finite cluster at $T=0$. For any fixed angle $\theta$ between the Mn-spins, assumed coplanar, the energy is found exactly. The ground state of this $p=0.5$ system is always at $\theta=0$ (FM-), while the energetically worse state is $\theta=\pi$ (AF-configuration). Their energy difference $\Delta E$ is a crude estimation of the FM state stability (Fig.2d). An optimal $J / t$ is found in all dimensions, with stability increasing with the coordination number [20]. The result Fig.2d is understood measuring the electronic density $n(i)$ of the same problem on a chain (Fig.3a). At small $J / t$, the delocalization manifests in the nearly uniform density, leading to weak FM. At large $J / t$, strong localization decouples the Mn-spins, producing again weak FM. However, there is an optimal value where the system takes advantage of $J / t$, but also allows for a nonzero effective coupling among separated classical spins, leading to a stronger FM.

Consider now the $x$ dependence of $T_{C}$. For simplicity, $J / t=2$ is mainly studied, which is both close to optimal and experimentally realistic [22]. Fig.3b shows $T_{C}$ vs. $x$ at $p \sim 0.4$, and for two reasonable values of $t$. Experiments [1] indicate a linear growth of $T_{C}$ up to $5 \%$ (shown), as in the numerical results. The slope of $T_{C}$ vs. $x$ is in remarkable agreement with $\mathrm{MC}$ predictions, in a reasonable range of $t$. Regarding $x>0.05$, a reduction of $T_{C}$ was originally reported in experiments [1]. However, recent data gathered with an optimized annealing treatment 23] indicate a $T_{C}$ "plateau". This still seems in contradiction with the linearly growing $T_{C}$ of the MC results, but it suggests that even more refined thin-films may continue increasing $T_{C}$ with increasing $x$. The MC results clearly indicate linear behavior up to $x \sim 0.25$ (Fig.3b). To
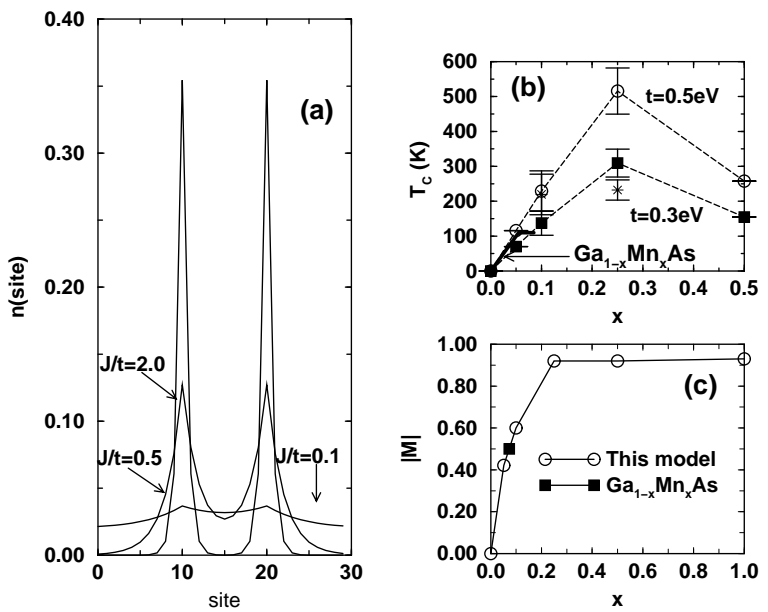

FIG. 3: (a) Exact $T=0$ local carrier density, for 1 carrier and 2 parallel spins at sites 10 and 20, of a 30-site chain, varying $J / t$. (b) $T_{C}$ vs. $x$ for $\mathrm{Ga}_{1-x} \mathrm{Mn}_{x}$ As [21] (thick line), and for model Eq.(1) using $t=0.3 \mathrm{eV}$ (squares) and $t=0.5 \mathrm{eV}$ (circles). In both cases, $p \sim 0.4$ and $J / t=2.0$. Typical error bars are shown. Stars are results for $p \sim 0.5, J / t=1$, and $t=0.5$ eV. (c) Magnetization $|M|$ vs. $x$ for model Eq.(1) (circles) at $T / t=0.005, J / t=2, p=0.4$, compared with the experimental value [1] for $\mathrm{Ga}_{1-x} \mathrm{Mn}_{x}$ As at $x \sim 0.07$ and $T=2 \mathrm{~K}$ (squares).

the extend that our model describes DMS quantitatively, higher values of $T_{C}$ could be expected experimentally. Regarding the presence of a $T_{C}$ maximum at $x=0.25$ : the origin of this effect is the growing probability with $x$ of having both holes and Mn-spins at NN-sites. In this case, AF links are formed since $J / t=2$ is not so strong to keep the link FM, reducing $T_{C}$ at large $x$ even at $p=0.5$. As $J / t$ grows, the effect diminishes and the maximum in $T_{C}$ moves toward $x=1$, as naively expected. Reciprocally, as $J / t$ decreases from $2(J / t=1$ shown in Fig.3b), the maximum in $T_{C}$ moves toward smaller $x$ 's, and only $t>0.5$ can provide high- $T$ ferromagnetism. This illustrates the key role that the optimization of $J / t$ plays in these models, effect not captured by MF approximations.

$|M|$ at $T \sim 0$ is in Fig.3c. In agreement with experiments, the $x \sim 0.1$ result indicates a magnetization $\sim 50 \%$ of its maximum value. This nonsaturated behavior originates in the random distribution of Mn-spins, since Mnclusters are formed providing a trap to holes. Nonclustered spins are not much visited by those holes, and their spins are not polarized. With growing $x$, holes are more itinerant, polarizing the entire sample (Fig.3c) [24].

In summary, MC investigations of a spin-fermion model for DMS unveils substantial differences with previously reported results employing MF techniques. The subtle regime of intermediate $J / t$ appears the most relevant in these compounds. $T_{C} \sim 0.08 t$ is an upper limit for the FM critical temperature, result close to those accepted for $x=1$ [4, 25]. Our main results are summarized in Fig.4, that contain $(\mathrm{a}, \mathrm{b})$ the nontrivial $J / t$ dependence of $T_{C}$ and $T^{*}$, and (c) $T_{C}$ with varying $x$ and $p$, at optimal $J / t$. To the extend that the present model is ap- 
(a)
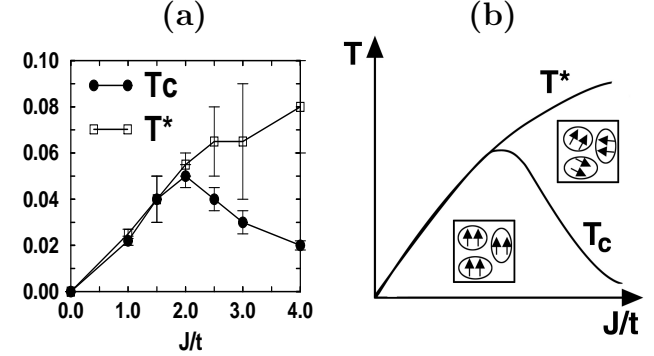

(c)

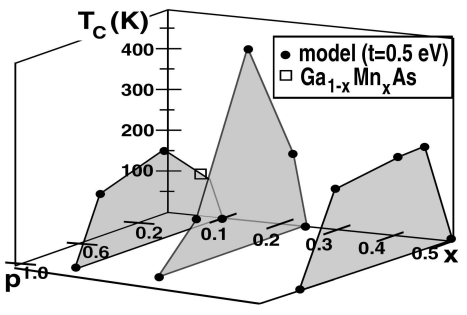

FIG. 4: (a) MC phase diagram in $2 \mathrm{D}$ varying $J / t$, at fixed $x$ and $p$. At large $J / t$, a broad scale $T^{*}$ corresponds to the formation of uncorrelated clusters. $T_{C}$ is the "true" transition temperature, defined as the $T$ where FM correlations develop at the largest distance in the clusters used. At small $J / t$, those temperatures are similar. The optimal $J / t$ is intermediate between itinerant and localized regimes. (b) Schematic phase diagram believed to be valid both in $2 \mathrm{D}$ and $3 \mathrm{D}$, with the clustered and FM states indicated. (c) Numerically obtained $T_{C}$ vs. $x$ and $p$, at $J / t=2.0$. Filled circles are from model Eq.(1) with $t=0.5 \mathrm{eV}$. The open square corresponds to the experimental value for $\mathrm{Ga}_{1-x} \mathrm{Mn}_{x}$ As at $x \sim 0.1$. plicable to DMS materials, broad guidelines to improve $T_{C}$ can be established: (i) The optimal $J /\left.t\right|_{o p t} \sim 2$ must be intermediate between the itinerant and localized limits (Fig.4a,b). This $J / t$, or larger, is expected to keep the semiconducting nature of the state at $T>T_{C}$. Only band calculations beyond our model can predict which particular material will have such an optimal $J / t$. (ii) $x$ should be increased beyond 0.1 . At $J /\left.t\right|_{\text {opt }}$, the best value is $x \sim 0.25$. Currently, $x=0.14$ is the experimental limit [26]. (iii) The number of antisite defects must be controlled such that $p \sim 0.5$ ( $p \sim 1$ would be detrimental due to competing antiferromagnetism). (iv) As the coordination number grows, $T_{C}$ grows. (v) Finally, the simplest procedure to increase $T_{C}$ relies on increasing the scale $t$. In fact, $(\mathrm{Ga}, \mathrm{Mn}) \mathrm{As}$ and $(\mathrm{In}, \mathrm{Mn}) \mathrm{As}$ have different hybridization strengths [27], and this should be an important consideration in studying new materials. Our work also suggests formal analogies between DMS and manganite models, with similar $T_{C}$ 's, and a related clustered state above ordering temperatures.

Work supported by NSF grant DMR-0122523 and by MARTECH.
[1] H. Ohno, Science, 281, 951 (1998).

[2] T. Dietl, cond-mat/0201282.

[3] K. Yu et al., Phys. Rev. B65, 201303 (2002).

[4] E. Dagotto et al., Phys. Reports 344, 1 (2001).

[5] J. Schliemann and A. H. MacDonald, Phys. Rev. Lett. 88, 137201 (2002); J. Schliemann et al., Phys. Rev. B64, 165201 (2001); Appl. Phys. Lett. 78, 1550 (2001). Hybrid $\mathrm{MC}$ studies in the latter are carried out in a continuum formulation.

[6] M. Berciu and R. N. Bhatt, Phys. Rev. Lett. 87, 107203 (2001); M. P. Kennett et al. . cond-mat/0203173. See also C. Timm et al., cond-mat/0111504.

[7] A. Chattopadhyay et al., Phys. Rev. Lett. 87, 227202 (2001).

[8] A. Kaminski and S. Das Sarma, Phys. Rev. Lett. 88, 247202 (2002).

[9] M. Mayr et al., Phys. Rev. B65, 241202 (RC) (2002).

[10] M. J. Calderón et al., cond-mat/0203404.

[11] Our study shows the standard $T_{C}$ increase with coordination number. If a large $T_{C}$ is reached with cubic, even larger values are expected with realistic FCC lattices.

[12] This assumption is much used in Mn-oxide studies, where a $S=3 / 2 t_{2 g}$-spin is usually considered classical 4 .

[13] J. Okabayashi et al., Phys. Rev. B58, 4211 (1998).

[14] S. Sanvito et al., Phys. Rev. B63, 165206 (2001).

[15] Potential disorder is neglected [8, 10] (to be included in future publications).

[16] N. Furukawa et al., Comp. Phys. Comm. 142, 410 (2001).
[17] Our results for $x=0.1, p=0.1, J / t=2$, with a $T_{C} / t \sim 0.02$ for an $8^{3}$ cluster, are comparable with results of Ref. 10 at $x=0.1, p=0.07, J / t=3$, using a $14^{3}$ cluster, that report $T_{C} / t \sim 0.03$. This reasonable agreement in the difficult regime of small $x$ and $p$ suggests that size effects are under reasonable control in these studies.

[18] J. L. Alonso et al., Nucl. Phys. B 596, 587 (2001).

[19] The existence of a $T^{*}$ is of much interest in manganites, where the state between $T_{C}$ and $T^{*}$ is believed to cause the colossal magnetoresistance (see J. Burgy et al., Phys. Rev. Lett. 87, 277202 (2001)). Other doped magnetic systems, such as $\mathrm{Eu}_{1-x} \mathrm{Gd}_{x} \mathrm{O}$, have a similar phenomenology (H. Rho et al., Phys. Rev. Lett. 88, 127401 (2002)).

[20] The optimal $J / t$ of this "toy" example is larger only by a factor 2, compared with the realistic value Fig.2c.

[21] S. J. Potashnik et al., Appl. Phys. Lett. 791495 (2001).

[22] For $J / t \gg 1$ values, the optimal density for $T_{C}$ would be $x=1$, as in manganites.

[23] S. J. Potashnik et al., cond-mat/0204250.

[24] Note also that the nonsaturated low- $T$ state can be fully polarized with relatively small magnetic fields: in our studies, fields of just $10 \mathrm{~T}$ are sufficient to raise $|M|$ from 0.5 to nearly 1.0 , at $x=0.1$.

[25] $\mathrm{La}_{0.6} \mathrm{Sr}_{0.4} \mathrm{MnO}_{3}$ has a $T_{C} \sim 340 \mathrm{~K}$, and our simulations suggest that similar values could be reached for DMS.

[26] A. J. Blattner and B. W. Wessels, cond-mat/0205602.

[27] J. Okabayashi et al., cond-mat/0203056. 